\title{
The role of mathematical models in explaining recurrent outbreaks of infectious childhood diseases
}

First/Middle Name: Chris T.

Surname: Bauch

Academic Title: Associate Professor

Unit: Department of Mathematics and Statistics

University: University of Guelph

Street Name: Stone Road East

Street Number: 50

Postal Code: N1G 2W1

City: Guelph

Province: Ontario

Country: Canada

email: cbauch@uoguelph.ca

Homepage URL: http://www.uoguelph.ca/ ${ }^{\sim}$ bauch

Country Code/Telephone Number: +1 519-824-4120 ext. 53079

Country Code/Fax Number: +1 519-837-0221

Summary. Infectious childhood diseases such as measles are characterized by recurrent outbreaks. Mathematicians have long used models in an effort to better understand and predict these recurrent outbreak patterns. This paper summarizes and comments upon those efforts, providing a historical outline of childhood disease models that have been developed since the start of the twentieth century. This paper also discusses the influence of data analysis techniques, such as spectral analysis, on the understanding and modelling of childhood disease dynamics.

\section{Introduction}

Childhood diseases (such as measles, whooping cough, chickenpox, polio, mumps and rubella) have had major and longstanding impacts on public health. Although vaccination programmes have significantly reduced morbidity and mortality from many childhood diseases [1], and are close to eradicating polio altogether [2], to date only smallpox has been completely eradicated through vaccination $[3,4]$. Moreover, measles continues to kill hundreds of 
thousands of undernourished and unvaccinated children in lesser-developed countries each year [5]. In the face of persistent vaccine scares that often cause a resurgence in childhood diseases $[6,7,8]$, an understanding of childhood disease transmission and control remains essential.

The mathematical modelling of childhood diseases continues to be an area of active research. This is due to the significant morbidity and mortality associated with childhood diseases, their relatively simple epidemiology, and the widespread availability of time series data describing childhood disease incidence (number of new cases per unit time). Time series of childhood disease incidence are the most detailed and complete ecological time series available for any organism, and therefore also provide a way of testing ecological theory $[9,10]$.

Outbreak patterns for childhood diseases are always recurrent, such that peaks in disease incidence alternate with troughs. However, there is much variation in outbreak patterns across diseases, places and times. Some time series exhibit highly regular annual or biennial outbreaks, some exhibit much longer interepidemic intervals, while others exhibit apparently irregular outbreaks (Figure 1a-d) [11, 12]. Outbreak patterns for a given disease can also change over time, either gradually or through abrupt transitions (Figure 1a,c,d) [11, 12]. One of the goals of mathematical epidemiology during the twentieth century has been to determine the causes of recurrent outbreaks of childhood diseases and predict the interepidemic interval, defined as the time between successive outbreaks [13].

Immunity to childhood diseases is generally lifelong, or long-term (however in the case of whooping cough there may be significant asymptomatic transmission among adults [16]). Susceptible individuals gradually accumulate in the population through births, until there is a sufficient density of susceptibles for an epidemic to start. Epidemiological timescales are fast compared to demographic timescales, hence the epidemic rapidly depletes the susceptible pool and the epidemic eventually dies out. Susceptibles are gradually built up again through new births and the pattern recurs. If this accumulation of susceptibles occurs more slowly, for instance through a reduced birth rate or higher rates of vaccine uptake, then the interepidemic interval increases accordingly.

It is important to emphasize that this 'dissection' of an epidemic cycle is ultimately a description of recurrence, not an explanation (even though it is often presented as such). One could equally well imagine a scenario in which the influx of new susceptibles exactly balances the incidence of disease at all times, so that the susceptible pool is depleted at the same constant rate as it is refilled. This would imply a constant number of cases over time instead of recurrent outbreaks. Without modelling, it is not clear a priori why incidence should exhibit recurrence instead of approaching a stable equilibrium. Modelling is also essential to quantitatively predict the interepidemic interval, and the time of transition between outbreak patterns. Modelling is indispensable not only for understanding recurrence as a natural phenomenon but also 

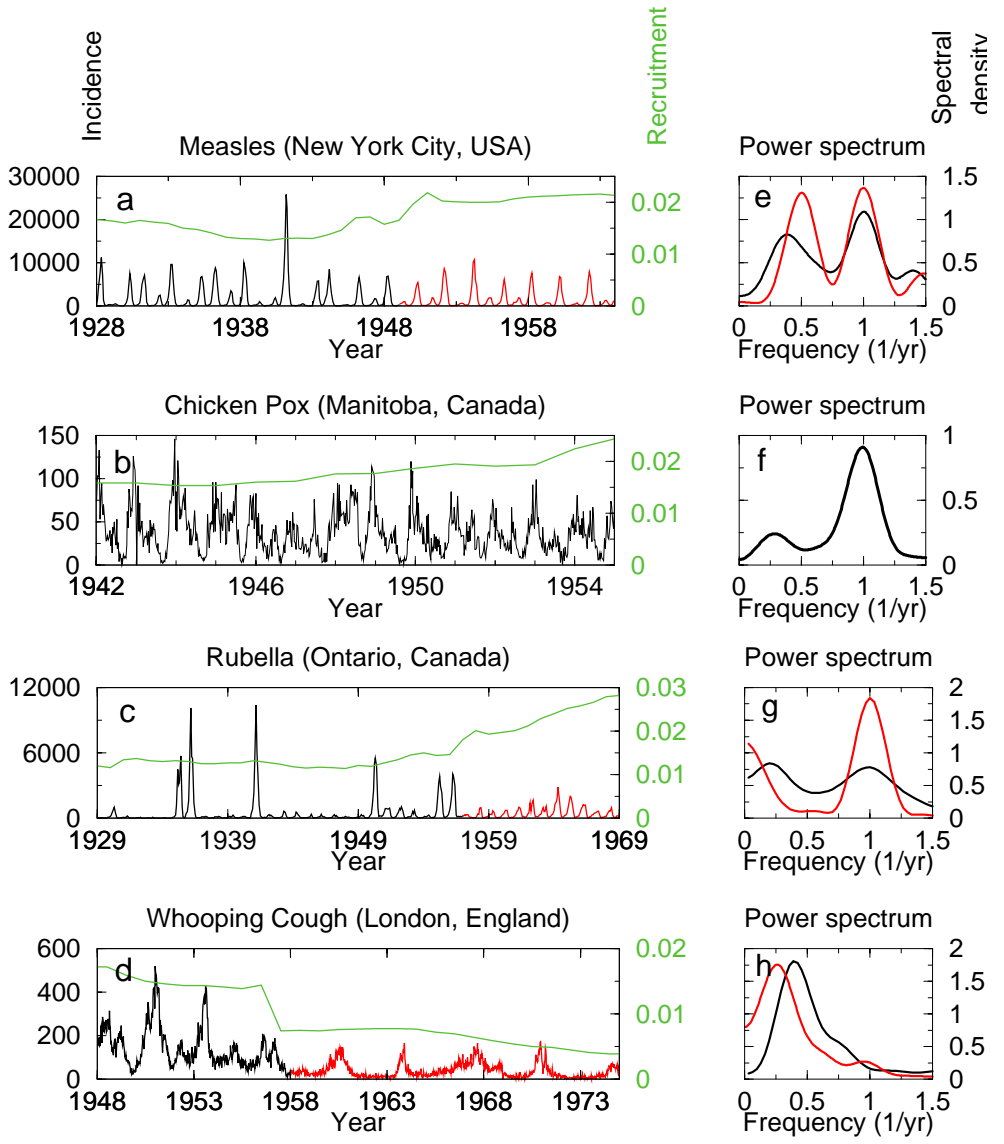

Fig. 1. Time series of incidence $(\mathrm{a}-\mathrm{d})$ and power spectra $(\mathrm{e}-\mathrm{h})$ for four common childhood diseases. In each panel, the green line shows annual susceptible recruitment, $\nu(1-p)$, where $\nu$ denotes births normalized by 1955 population size and $p$ denotes proportion vaccinated ( $c f$ Ref. [11]); recruitment is shown displaced forward in time by the mean age at infection to account for the typical delay between birth and infection (4 years for measles, 7 years for chickenpox, 11 years for rubella and 4 years for whooping cough). Time series are divided into sections based on substantial differences in recruitment rates; the corresponding power spectra are not sensitive to the precise point at which the time series is divided (the chickenpox time series has not been divided because no dramatic change in births occurred during the period covered by the data). The power spectrum is the Fourier transform of the autocovariance function of the time series $[14,15]$. Before computation of the power spectrum the data were trend-corrected and tapered with a double cosine bell [14]. The autocovariance function was smoothed with a Tukey window [14] to reduce variance in the power spectrum, which facilitates locating the spectral peaks. The width of the Tukey window was chosen so that the resulting bandwidth was the same (0.35) for both weekly and monthly incidence time series. Figure reproduced from Ref. [12]. 
for informing public health policy, since it allows one to predict how changes in vaccination coverage and demographic parameters alter the time between outbreaks. Indeed, age-structured measles models show good agreement with pre- and post-vaccination age-stratified case reports and seroprevalence surveys [17], and have been successfully used in this way to predict and plan for upcoming measles epidemics in New Zealand, for example [18].

This paper reviews the role of models in understanding and predicting recurrent outbreak patterns of childhood diseases. Section 2 describes the SIR model with demographics (a classic epidemic model applicable to childhood diseases). Section 3 describes the historical development of mathematical models used to study recurrence in childhood diseases, and Section 4 describes the influence of data analysis techniques on our understanding of recurrence. Concluding comments are made in Section 5.

\section{The SIR model with demographics}

Compartmental models allocate individuals into mutually exclusive categories (compartments) based on infection status, age, social group, or other categories of interest or epidemiological relevance. If a sufficiently large population is assumed, then differential equations can be derived to describe the time evolution of the number of individuals in each compartment. Alternatively, a stochastic (e.g. Monte Carlo or individual-based) model can also be simulated or analyzed to study the time evolution of the compartments, particularly when the population size is small.

A classic model for childhood diseases is the SIR model with demographics, which assigns compartments for the number of susceptible $(S)$, infected $(I)$, and recovered $(R)$ individuals (Figure 2). For modelling an endemic disease, birth and death are important, and so individuals in each compartment are assumed to give birth at constant per capita rate $\nu$ (all newborns are susceptible), and individuals in each compartment die at constant per capita rate $\mu$ (disease-related mortality is neglected). Infected individuals recover at a constant per capita rate $\gamma$ and retain lifelong immunity. Finally, we assume that the incidence is proportional to the product of the number of susceptible and infected individuals (new cases arise through mass-action mixing between susceptible and infected individuals). These assumptions yield the system of ordinary differential equations

$$
\begin{aligned}
\dot{S} & =\nu N-\beta I S-\mu S, \\
\dot{I} & =\beta I S-\gamma I-\mu I, \\
\dot{R} & =\gamma I-\mu R .
\end{aligned}
$$

where $N=S+I+R$ is the population size, $\beta$ is the mean rate at which an infected individual transmits the infection to a susceptible, and other parameters are as defined above. Note that $1 / \gamma$ is the mean duration of infectiousness. 
It is often assumed that $\nu=\mu$, such that $S+I+R=N$ and the population size is constant. Then, the population size $N$ can be normalized to 1 for simplicity of notation (hence $S, I$ and $R$ become densities). Under these conditions, for $\beta /(\gamma+\mu) \leq 1$, the equations exhibit a disease-free equilibrium $(1,0,0)$ that is globally asymptotically stable. Conversely, when $\beta /(\gamma+\mu)>1$, the disease-free equilibrium is unstable and there is a globally asymptotically stable endemic equilibrium $\left(\frac{\gamma+\mu}{\beta}, \frac{\mu(\beta-\gamma-\mu)}{\beta(\gamma+\mu)}, \frac{\gamma(\beta-\gamma-\mu)}{\beta(\gamma+\mu)}\right)$. Although this model ignores many aspects of real-world demography and epidemiology, it is pedagogically useful nonetheless, and we will see how it quantitatively captures certain features of real epidemics despite its simplifications.

The SEIR model with demographics is an extension of the SIR model that is also applied to childhood diseases. The ' $\mathrm{E}$ ' compartment is a latent stage where individuals are infected but not yet infectious. Individuals leave the latent compartment ' $\mathrm{E}$ ' and enter the infectious compartment ' $\mathrm{I}$ ' at some constant rate, the inverse of which equals the mean latent period. Latency is common in childhood diseases.

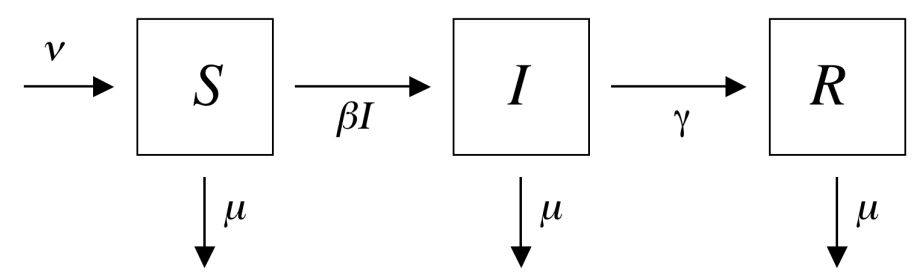

Fig. 2. Diagram of SIR model with demographics (Eqs. 1-3). Terms next to arrows denote per capita rates of transitions into or out of compartments.

The basic reproductive number, $\mathcal{R}_{0}$, is the average number of secondary infections produced by a typical infected individual in a fully susceptible population $[19,20]$. When $\mathcal{R}_{0}>1$, each infected case more than replaces itself, and incidence grows geometrically, causing an epidemic. However, stochastic effects can cause the disease to fade out in the early stages, even when $\mathcal{R}_{0}>1$ (since the number of infected individuals is initially very small). For childhood diseases, $\mathcal{R}_{0}$ varies widely depending upon the disease, location, and time. In most Western countries during the $20^{\text {th }}$ century, $\mathcal{R}_{0}$ was typically highest for measles and whooping cough $\left(\mathcal{R}_{0} \approx 11-18\right)$, in the middle range for chickenpox and mumps $\left(\mathcal{R}_{0} \approx 7-14\right)$, and lowest for rubella $\left(\mathcal{R}_{0} \approx 6-9\right)$ [19]. For the SIR model with demographics (Eqs. 1-3), it can be shown that $\mathcal{R}_{0}=\beta /(\gamma+\mu)$, which is the inverse of the proportion susceptible at the endemic equilibrium.

An accurate model of recurrent outbreaks would be expected to exhibit sustained oscillations (e.g. stable limit cycles). However, stability analysis of the endemic equilibrium of the SIR model shows that it is a stable spiral, therefore solutions converge to it via damped oscillations with period 


$$
T \approx 2 \pi \sqrt{\frac{1}{\gamma \mu\left(\mathcal{R}_{0}-1\right)}} .
$$

Therefore, the simple SIR model alone cannot account for the recurrent outbreaks observed in incidence time series.

However, a number of extensions to the basic SIR model have been found to generate sustained oscillations. These extensions fall into two categories. In first category of endogenous mechanisms, the period of oscillation is not explicitly incorporated as a model parameter, but rather it is implicit in how the model is defined and its parameter values set. Endogenous mechanisms usually yield sustained oscillations by destabilizing the endemic equilibrium of the basic model (Eqs 1-3), giving rise to stable limit cycles with a period of approximately $T[21,22,23,24,25,26,27,28,29,19,30,31,32,33,34,35,36$, 37]. In contrast, in the second category of exogenous mechanisms, oscillations are produced through periodic (seasonal) forcing of model parameters such as the transmission rate $[21,38,39,40,25,28,11,37,12]$. The period of forcing is set explicitly as a model parameter, and the resulting periods of oscillation are simply integer multiples of the period of forcing.

Throughout the twentieth century, modellers have experimented with various extensions to the basic SIR model in an attempt to better account for the observed patterns of recurrent outbreaks, using endogenous and/or exogenous mechanisms. We outline this historical development in the next section.

\section{Historical development of compartmental models}

The sources for this historical review include both primary and secondary sources $[41,42,19,43,44,45]$. The epidemic modelling literature has become vast, especially in the past thirty years, and modellers have approached the problem of recurrence from many different angles. Indeed, this review itself constitutes just one angle from which the literature can be approached, and is not meant to be comprehensive.

\subsection{Early Models}

Daniel Bernoulli developed what was probably the first compartmental epidemic model in 1760 [46, 47, 48]. His model divided the population into susceptible and immune compartments and assumed an age-specific force of infection and case fatality rate, yielding a system of equations with an endemic equilibrium of susceptible and immune individuals. Bernoulli was not specifically interested in the cause of recurrent outbreaks or in predicting the interepidemic interval. Rather, his motivation was to predict the expected gain in

life expectancy that would be brought about by applying smallpox control measures. Since variolation was becoming widespread in Europe in the late 
1700s, predicting the resulting increase in life expectancy would have been important for pricing annuities [48].

For the next hundred years, apparently little work was done in epidemiological modelling. However in the mid-nineteenth century to the early twentieth century, the subject was broached again by a number of authors. During this time, papers were written on mathematical and statistical models for various types of infectious diseases $[49,50,51,52,53,54,55,21,56,57]$ but here we focus specifically on mathematical (including stochastic) models for childhood diseases.

In the nineteenth century, researchers were already seeking the causes of recurrent outbreaks. At this time it was already suspected that the density of susceptibles was an important quantity. As A. Hirsch claimed in 1883, 'the recurrence of the epidemics of measles at one particular place is connected neither with an unknown something (the mystical number of the Pythagoreans), nor with "general constitutional vicissitudes", as Köstlin thinks; but it depends solely on two factors, the time of importation of the morbid poison, and the number of persons susceptible of it' [58] (quoted from Ref. [21]).

However it was probably not until the early 1900s that researchers began using mechanistic models to explain and predict recurrent outbreaks in childhood diseases. At this time, there were at least two competing hypotheses regarding the causes of recurrence. Scientists such as Brownlee hypothesized that seasonal recurrence in diseases such as measles was simply due to seasonal variation in pathogen virulence [51]. By comparison, scientists such as W.H. Hamer and A. Davidson sought an endogenous explanation for recurrence. They suggested that it is unnecessary to invoke seasonal variation in host or pathogen properties, rather, a 'mechanical theory of numbers and density' would suffice [50]. More specifically, Hamer hypothesized in 1906 that incidence is proportional to the product of the densities of susceptible and infected individuals. This is now called the mass-action mixing assumption, and is a cornerstone of epidemic modelling.

Hamer geometrically analyzed an average measles epidemic curve to support this hypothesis (Figure 3). He also used this line of analysis to show that an epidemic can die out before all susceptibles have been depleted. Ultimately Hamer's hypothesis was found to be lacking [21], for the same reasons that the SIR model with demographics only exhibits damped oscillations (namely, mass-action mixing alone cannot result in sustained oscillations in models). However, in the context of what was known at the time, and without the benefit of developments in nonlinear mathematics, Hamer's hypothesis is arguably both subtle and original.

Hamer developed a discrete time model, but it was formulated numerically (without the aid of symbolic notation) and hence it was not readily recognizable as such. Epidemic models were established in a more recognizable, modern form through the seminal work of Kermack and McKendrick in the late 1920s [55, 56, 57] (reprinted in Ref. [59]). They showed that the density of susceptible individuals must exceed a critical threshold in order for 


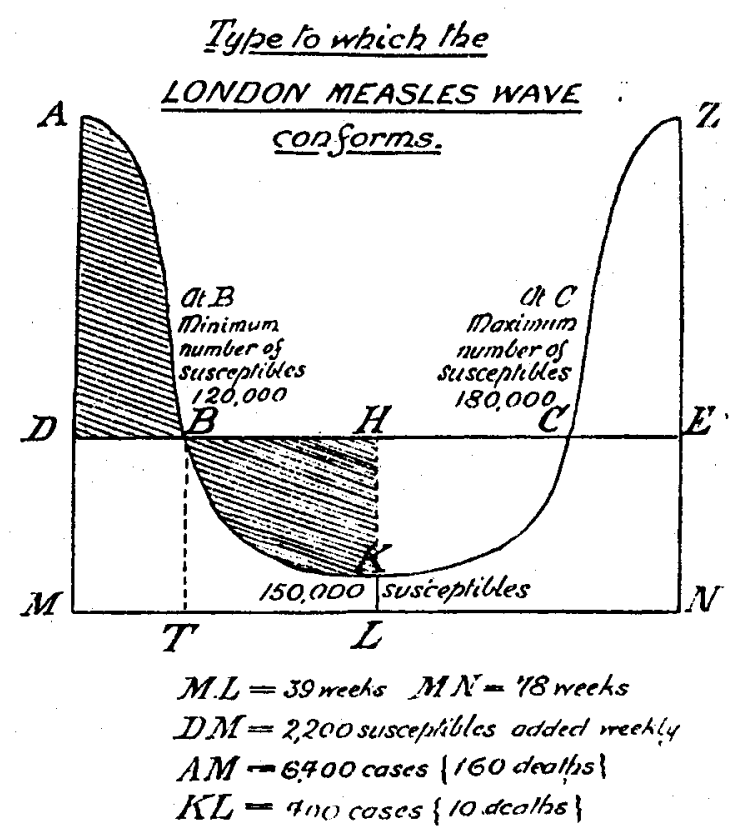

Fig. 3. Diagram representing an average measles epidemic curve in London, analyzed in Hamer's 1906 paper (Ref. [50]).

an epidemic to occur. (This result was also discovered by Ross in the early 1900s in the context of discrete-time malaria models [53]. It is also closely related to Hamer's finding that the susceptible pool is not fully depleted by the end of a natural epidemic curve.)

From general assumptions, Kermack and McKendrick derived an integrodifferential equation to describe the spread of an epidemic in a closed population,

$$
\dot{S}(t)=S(t) \int_{0}^{\infty} \bar{A}(\tau) \frac{d}{d t} S(t-\tau) d \tau,
$$

where $\bar{A}(\tau)$ is the expected infectivity of an individual who became infected a time $\tau$ ago, and $S(t)$ is the (spatial) density of susceptible individuals [55, 43]. For the special case that the $\bar{A}(\tau)=\beta \exp (-\gamma \tau)$, and by defining $I(t) \equiv$ $-\frac{1}{\beta} \int_{0}^{\infty} \bar{A}(\tau) \dot{S}(t-\tau) d \tau=-\frac{1}{\beta} \int_{-\infty}^{t} \bar{A}(t-\tau) \dot{S}(\tau) d \tau$ and differentiating, one recovers the SIR model without demographics $(\nu=\mu=0$ in Eqs. 1-3) [43].

Soon thereafter (in 1929) H.E. Soper, who felt he was 'merely following up the trail blazed by Sir William Hamer more than twenty years ago' [21], formulated and analyzed a discrete-time compartmental model to determine whether mass-action mixing was sufficient to generate sustained oscillations [21]. His model did generate sustained oscillations, however it was necessary to assume that all of an individual's infectiousness is concentrated at a single 
point in time at the end of the incubation period. In reality, of course, infectivity is distributed over some interval of time. Soper found that for a model with distributed infectivity, oscillations are damped and converged to an endemic equilibrium. As noted in Section 2, this is also true for the equivalent ordinary differential equation compartmental model (the SIR model with demographics), where the duration of infectiousness is exponentially distributed (Figure 4, top panel).
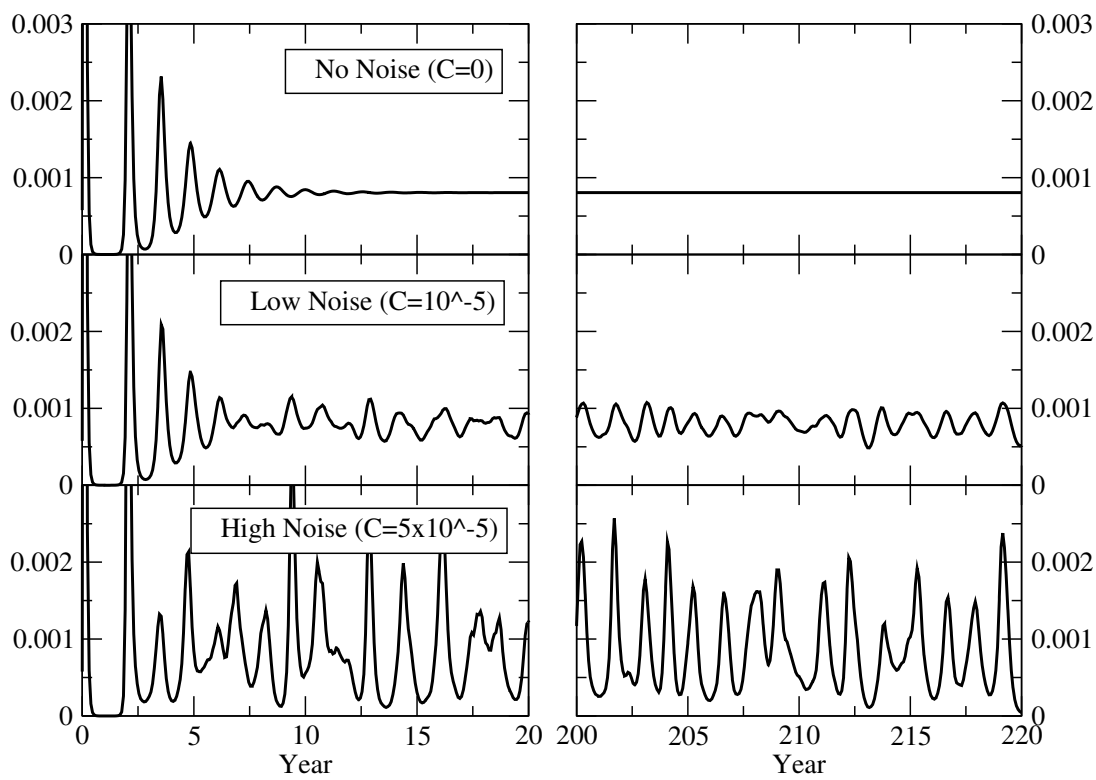

Fig. 4. The role of demographic stochasticity in sustaining oscillations. Panels from top to bottom show the effects of gradually increasing the amount of demographic stochasticity on time series of the density of infecteds, $I(t)$. A Gaussian (white) noise term was added to the birth and death parameters $\mu$ and $\nu$ in Eqs. 1-3: the value $C$ in the figure legends is the value of the coefficient of the normalized Gaussian noise term. Other parameters are $\beta=1240$ year $^{-1}, 1 / \gamma=15$ days, $1 / \mu=1 / \nu=50$ years.

Although Soper's model failed to produce sustained oscillations except under highly restrictive assumptions, his research stimulated further research on the problem [45]. One of the goals of mathematical epidemiology in the following decades was to correct for this apparent deficiency in Hamer-Soper models 
by introducing various extensions that would result in sustained oscillations. We review these extensions in the following subsections.

\subsection{Stochasticity}

Epidemic modelling started growing substantially in the 1950s [44]. During this time, the challenge of explaining recurrence was taken up by M.S. Bartlett, who took an entirely different (stochastic) approach to the problem $[22,60]$. Bartlett hypothesized that Hamer-Soper models failed to produce sustained outbreaks because they did not incorporate demographic stochasticity (stochastic effects associated with the discrete nature of births, deaths, immigration and emigration processes). These effects are present to some extent in all populations, but are especially significant for smaller population sizes.

Bartlett, using a stochastic model of measles transmission, showed that demographic stochasticity could sustain oscillations that would otherwise be damped. (This can also be seen by adding noise terms to the deterministic SIR model with demographics (Figure 4).) Bartlett also showed that the interepidemic interval of the oscillations in silico agreed approximately with the empirically observed values of the interepidemic interval [22,61]. It was found more recently (in 1998) that environmental stochasticity (stochasticity associated with environmental effects that affect the entire population at once, such as climatic fluctuations) also results in sustained oscillations in a model for pertussis [33].

\subsection{Seasonality}

Seasonality is a prominent feature in many incidence time series, with incidence for some diseases peaking consistently in the winter months (Figure 1a-d). Measles and chickenpox in particular are strongly seasonal. A number of driving forces have been hypothesized to be behind seasonal recurrence [62]. For instance, the seasonal structure of the school calendar means that transmission rates may be higher in Fall and Winter when school is in session (and children are crowded together) but lower during the summer holiday $[21,63]$. Likewise, seasonal variation in host physiology, pathogen virulence, and humidity/rainfall patterns may also play a role [62]. The relative importance of these factors is still unestablished, although evidence indicates that a seasonally-structured school calendar is at least partly responsible [63, 64].

Soper suspected seasonal variation in transmission rates might be important for explaining biennial measles outbreaks, and analyzed a variant of his discrete-time model with a seasonally-varying transmission rate [21]. However, more intensive research on seasonally-forced epidemic models did not begin until the 1970s $[38,25]$. Modellers often incorporate seasonality by making the transmission rate $\beta$ a sinusoidal function of time, e.g. $\beta(t)=\beta_{0}(1+\alpha \cos (2 \pi t))$

[40]. Some modellers take $\beta$ to be a step function based explicitly on real school 
calendars $[25,11,12]$. A seasonally-varying transmission rate yields oscillations at periods that are integer multiples of the period of forcing $[40,11,12]$.

\subsection{Age structure}

Susceptibility, infectivity, morbidity, and mortality rates often vary with age, and available data for these quantities are often broken down into age classes. Age-structured models have been the focus of many studies, and modellers have developed both continuous age-structured (PDE) and discrete agestructured (ODE) models, as well as stochastic and deterministic versions $[29,19,32,26,44,30]$. Moreover, other features such as seasonality [25, 28], stochasticity [65], and strain structure [27] have also been incorporated.

Basic age-structured compartmental models of the type appropriate for endemic childhood diseases, such as the continuous age-structured MSEIR model with demographics (where the ' $\mathrm{M}$ ' compartment is for maternally-derived immunity) [44], or the continuous age-structured SIR model with demographics $[29,19]$, either do not exhibit sustained oscillations at all (the endemic equilibrium being stable at all parameter values) [19, 29], or exhibit sustained oscillations only under highly restrictive assumptions [44,30]. Hence, age structure alone is apparently not sufficient to explain recurrence. However, oscillations in an age-structured models are typically very weakly damped [19, 29, 27], and so age structure at least facilitates recurrence. For oscillations to be sustained in an age-structured model, additional features must be incorporated, such as seasonality [25, 28], stochasticity [65] or strain structure [27].

\subsection{Alternative assumptions about incidence terms}

Conventionally, it is assumed that new cases are generated through homogeneous mixing, yielding the mass-action incidence term $\beta I S$ (Eqs. 1, 2), or the standard-incidence term $\beta I S / N$. The assumption of homogeneous mixing may be inaccurate, particularly under certain circumstances. Examples where the incidence does not depend linearly on the number of currently infected individuals include situations where a larger density of infected individuals decreases their per capita infectivity (saturation effects), and situations where multiple exposures to an infected individual are required for a transmission event to occur (threshold effects). Some researchers have shown that abandoning the conventional forms for the incidence term in the model equations can induce sustained oscillations [66, 67, 68, 69, 70].

For example, it has been argued that a more realistic term for incidence in many situations would be $\beta I^{p} S^{q} / N[69,70]$. The case $p>1$ corresponds to synergistic effects among pathogens, and may occur when viral concentration in the environment must exceed some critical threshold for transmission to occur (if the viral lifespan outside the host is short), or, for vector-borne diseases, when the disease vector needs to attack multiple infected hosts to attain sufficiently high viraemia for transmission to susceptible hosts to occur. 
When $p>1$, compartmental models can exhibit sustained oscillations at certain parameter values $[69,70]$.

\subsection{Distribution of latent and infectious period}

It is conventionally assumed that the latent and infectious periods (i.e. the duration of latency and infectiousness respectively) are distributed exponentially, such that the rate at which a latent individual becomes infectious, or the rate at which an infectious individuals recovers, is constant and independent of how long the individual has been in that compartment. Although this assumption yields relatively tractable ODEs, it is less realistic than other assumptions such as fixed or normally distributed durations.

If a fixed duration of infectiousness (incorporated through a time delay) is used instead, the endemic equilibrium of the basic SIR model can be destabilized through a Hopf bifurcation, yielding stable limit cycles [23, 24, 31, 34]. The resulting interepidemic interval is approximately the same as that predicted by local stability analysis of endemic equilibria of the corresponding models with an exponentially distributed periods (Eqn. 4). Results for more general assumptions on the distribution of latent and infectious periods have also been obtained [35, 36].

\subsection{Seasonality versus nonseasonality}

The mechanisms described in the previous subsections are not mutually exclusive, and it is most likely the case that multiple factors contribute to recurrence. It is difficult to determine the relative importance of these factors, however the fact that the time series exhibit two distinct types of outbreak patterns suggests the existence of at least two distinct mechanisms: some time series are characteristically seasonal (e.g. measles, chickenpox), whereas others are characteristically nonseasonal (e.g. whooping cough).

Models with seasonally-varying transmission rates can predict the interepidemic interval for diseases that are characteristically seasonal [38, 64, 11, 12], but they perform poorly for childhood diseases that are characteristically nonseasonal. For instance, seasonally-forced models predict that whooping cough should have an interepidemic interval of 1 year instead of the observed $\approx 4$ years $[71,72,12]$. Conversely, the period of damped oscillations $T$ computed from the basic model (Eqn. 4) or other models that use only endogenous mechanisms predicts the observed interepidemic interval for diseases that are characteristically nonseasonal [22, 61, 15, 19], but cannot predict seasonal outbreaks. Furthermore, for some diseases, the error of the latter models is enormous: for chickenpox, which is highly seasonal, the observed interepidemic interval in almost all cities is 1 year (e.g. Figure 1b), but models without seasonal forcing predict $T \approx 3[19,12]$.

Using a seasonally-forced SEIR model, it has been demonstrated numerically that solutions for measles remain close to the attractor (a biennial cycle) 
in the presence of demographic stochasticity, whereas solutions for whooping cough stray far from the attractor (an annual cycle), resulting in $\mathrm{a} \approx 4$ year interepidemic interval $[72,71,12]$. Hence, the relative importance of deterministic (seasonal) versus stochastic (nonseasonal) effects for these two diseases may explain the observed differences between their outbreak patterns. Indeed, a perturbative analysis of the attractors of a seasonally-forced model allows one to predict both seasonal and nonseasonal oscillations in measles, whooping cough, chickenpox and rubella [12]. Additionally, Monte Carlo simulations indicate some of the factors which determine the relative importance of seasonal and nonseasonal effects for a given disease [12]. Seasonality versus nonseasonality in incidence time series will be discussed more fully in the section on spectral analysis (Section 4).

\subsection{Chaos}

Mathematicians have long sought to determine whether chaos (sensitive dependence upon initial conditions) exists in natural and laboratory populations [73]. Because time series of childhood disease incidence are relatively long and accurate (in comparison to many ecological time series), researchers have devoted significant attention to seeking chaos in childhood disease dynamics, particularly for measles $[74,75,76,77,78,79,80,81,82]$. The presence or absence of chaos in disease dynamics does not bear directly on the question of what causes recurrent outbreaks, however, the issue has much to do with characterizing outbreak patterns as well as with forecasting future outbreaks $[80,82]$.

Some researchers have attempted to detect the hallmarks of chaos in time series by computing Lyapunov exponents [75, 81], deriving return maps from reconstructed phase space trajectories [76], computing correlation dimensions [75], or applying nonlinear forecasting techniques [82, 80]. Other researchers have constructed epidemic models, pointing out parallels between model dynamics in chaotic parameter regimes and real-world time series. However, existing methods require data sets that are even more extensive than those currently available $[75,81]$, hence the evidence in support of chaos in measles time series remains equivocal.

\subsection{Transitions between outbreak patterns}

As noted in Section 1, incidence time series often exhibit sudden or gradual transitions between outbreak patterns for a given disease in a given population. Measles in New York City, USA, for example, exhibited a dramatic shift from an apparently irregular pattern to a regular biennial pattern in the mid 1940s (Figure 1a). Some researchers have suggested that these transitions, particularly for measles, are caused by endogenous mechanisms such as chaos [83] or noise-driven shifts between basins of coexisting attractors [40]. Other researchers have emphasized the impact of exogenous changes in parameters 
controlling birth rates and vaccine uptake (which move the system to a new set of attractors) and report quantitative agreement between incidence time series and seasonally-forced SEIR models $[11,12]$.

\section{Spectral analysis of incidence time series}

The focus of this review so far has been on using models to understand recurrence. In comparison, the present Section focuses on how data analysis can help researchers understand recurrence. Techniques of data analysis are a 'lens' through which a system can be observed. They can sharpen or clarify certain aspects of the data, and thus change our understanding of the system, and how the system is modelled.

An example of how data analysis can clarify understanding comes from considering the interepidemic interval, which is a convenient but potentially misleading concept. Many time series do not have a well-defined time between outbreaks, because the magnitude and timing of outbreaks can vary considerably. For instance, in Liverpool, England, 1950-1967, before the era of mass vaccination (Figure 5), close inspection reveals a complex pattern where small epidemics sometimes follow one another in rapid succession, on the order of months, and significant year-to-year variation in the magnitude of larger epidemics. The 'time between outbreaks' depends upon how large a peak needs to be before it is considered an outbreak. The best that can be said is that the time between outbreaks is, very roughly, 1-2 years. Likewise, for measles in New York City from 1944-1962, there exists a regular biennial pattern where small outbreaks alternate with large outbreaks (Figure 1a). For this time series, the interepidemic interval could be equally well-argued to be either one year or two years. Similar ambiguities can be seen for other diseases (Figure 1b-d). Hence, the interepidemic interval can be an imprecise and arbitrary concept.

\subsection{Power spectra}

Some of this ambiguity can be eliminated by applying spectral analysis to the time series [14, 84]. For instance, starting in the 1980s, epidemic modellers began computing power spectra of incidence time series [15, 19, 75, 37, 12]. The power spectrum of a time series describes the relative contributions from each frequency of oscillation to the time series, over some fixed time interval. A single dominant peak at a frequency of $1 / Y$ years $^{-1}$ in a power spectrum would therefore correspond to recurrent outbreaks every $Y$ years. Power spectra can be computed, for example, by de-trending the incidence time series, computing the Fourier transform of the autocorrelation function of the detrended time series, and then smoothing with a suitable spectral window such as a Tukey window or a Bartlett window [15, 19, 12]. Figures 1, 5 and 6 show power spectra of childhood disease incidence time series. 
Measles Incidence in Liverpool, England
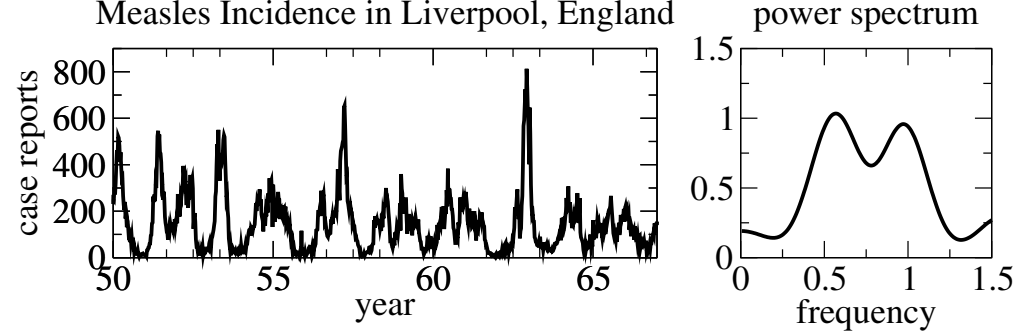

Measles Incidence in Ontario, Canada
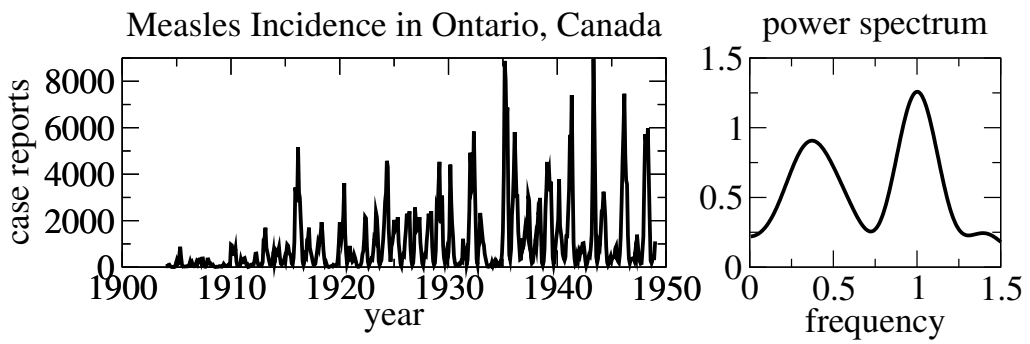

Fig. 5. Incidence of measles in Liverpool, England 1950-1967 (top left panel) and corresponding power spectrum (top right panel), and measles case reports in Ontario, Canada 1904-1948 (bottom left panel) and corresponding power spectrum (bottom right panel). See Figure 1 caption for details on computation of power spectra.

Power spectra can reveal much about a time series that is not obvious upon casual inspection. For instance, power spectra of time series of childhood disease incidence reveal that spectral peaks can be either seasonal (corresponding to seasonality in the time series, and occurring at frequencies $1 / n$ years $^{-1}$, where $n$ is an integer) or non-seasonal (corresponding to nonseasonality in the time series and occurring at any real-valued frequency) [12]. For New York City 1944-1962, spectral peaks appear at frequencies of 1 year $^{-1}$ and $1 / 2$ years $^{-1}$, corresponding to the biennium observed in the time series for those years (Figure 1a,e). Power spectra of childhood disease time series generally exhibit both seasonal and nonseasonal peaks, although their relative magnitude may differ significantly (Figures 1,5 and 6) [12]. For the apparently irregular time series of measles in Liverpool 1950-1967 (Figure 5) there is both a seasonal peak at a frequency of 1 year $^{-1}$ and a nonseasonal peak occurring at a frequency of $1 / 1.8$ years $^{-1}$. For measles in Ontario 1904-1948 (Figure 5) there is both a seasonal peak at 1 year $^{-1}$ and a nonseasonal peak at $1 / 2.7$ years $^{-1}$. Smaller populations exhibit larger nonseasonal spectral peaks, perhaps because stochastic effects are more important (see Subsection 3.7). 
This can be seen in chickenpox power spectra for five Canadian provinces with differing population sizes for the years 1942-1955 (Figure 6).

Seasonal peaks are associated with exogenous (seasonal) effects, such as seasonally-varying transmission rates, whereas nonseasonal peaks are associated with endogenous effects, such as stochasticity and other mechanisms discussed in Section 3. Power spectra provide a way of characterizing outbreak patterns that is less ambiguous than the interepidemic interval, since one can simply identify the frequencies and relative magnitudes of seasonal and nonseasonal peaks in the power spectrum. However, power spectra do not contain phase information, and therefore on their own they are insufficient to completely specify a time series. There is also a danger in choosing a bandwidth for the smoothing window that is too narrow, yielding spurious spectral peaks.

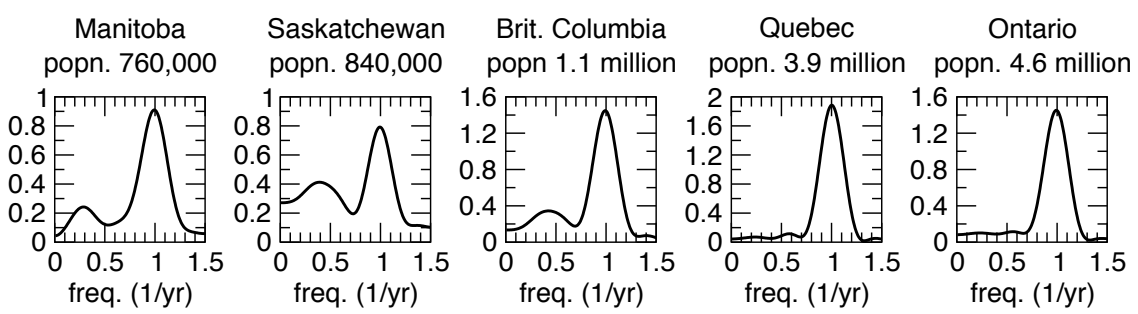

Fig. 6. Power spectra of chickenpox incidence time series in five Canadian provinces for the years 1942-1955, showing larger nonseasonal peaks for smaller population sizes. See Figure 1 caption for details of computation of power spectra.

Power spectral analysis illustrates how data analysis can provide new ways of describing and characterizing incidence time series. This, in turn, may lead to a better understanding of disease dynamics and may also influence modelling. For instance, the fact that spectral peaks can be classified into seasonal and non-seasonal types, and the fact that many spectra simultaneously exhibit both types of peaks, suggests that recurrence is not a monolithic phenomenon. Rather, there seem to be two distinct types of recurrence, each with its associated causes, and each of which is present to some extent in all childhood disease time series. Therefore it is important to incorporate multiple mechanisms into epidemic models, in cases where a 'true-to-life' representation of recurrence is desirable. Many epidemic models have focused separately on mechanisms such as seasonality [21], age-structure [29], or stochasticity [22]. In the past few decades, modellers have begun studying models with multiple mechanisms, such as seasonality and age structure [25, 28], stochasticity and seasonality $[71,72,12]$, or age structure and strain structure [27]. 


\subsection{Wavelet power spectra}

As demographic and epidemiological parameters evolve over time, the frequencies exhibited in the time series can also change. However, the method of computing power spectra as described above assumes that frequencies are stationary over the time interval for which the power spectrum is computed, and thus cannot capture these trends.

A method of spectral analysis that circumvents this problem is wavelet analysis [84]. Wavelet analysis also decomposes a time series into component frequencies, however because the method uses wavelets (which are localized in time), it provides a temporally localized description of component frequencies, i.e. it can describe the time evolution of component frequencies.

Wavelet analysis can be applied to childhood disease time series [85], and can reveal interesting temporal trends. For instance, in the wavelet power spectrum for New York City 1928-1966 (Figure 7) we observe a sudden shift from a non-seasonal period of approximately 2.5 years to a seasonal period of 2 years, a transition that was apparently induced by the baby-boom (Figure 1a) [11]. The suddenness of this transition is consistent with bifurcation analysis of a seasonally-forced SEIR model, which for these parameters predicts a shift from an annual attractor to a biennial attractor $[11,12]$. Likewise, in London, England 1944-1994, upon the initiation of mass vaccination, we observe a transition from a biennial pattern to a pattern of gradual increase in the non-seasonal period, as vaccine coverage increases over time (Figure 7). Such obvious patterns do not appear in all wavelet power spectra for childhood diseases, and they can sometimes be difficult to interpret (e.g. for measles in Ontario, Figure 7). Nonetheless, wavelet analysis can refine our understanding of incidence time series and the underlying disease dynamics.

\section{Conclusions}

This paper has reviewed how compartmental epidemic models have been used to understand and predict recurrent outbreaks of childhood diseases. The classic SIR model with demographics was presented, and its shortcomings with respect to explaining recurrence were discussed. The historical development of childhood disease models was then reviewed, with particular emphasis on efforts to correct this apparent deficiency of the classic SIR model, by developing and analyzing models that exhibit sustained oscillations through various mechanisms. The paper concluded with a discussion of the impact of spectral analysis techniques on our understanding of childhood disease dynamics.

Childhood disease modelling has received sustained interest over many decades. Generally speaking, these models, and the methods by which they are analyzed, have become increasingly diverse and sophisticated. Perhaps one reason for this sustained interested and continuing development has been a series of partial successes: models often successfully explain certain features 


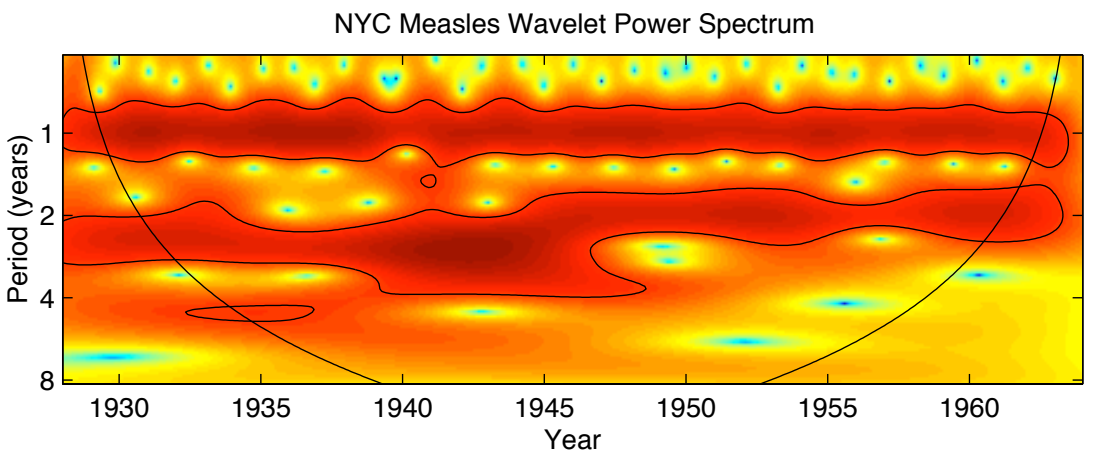

London Measles Wavelet Power Spectrum
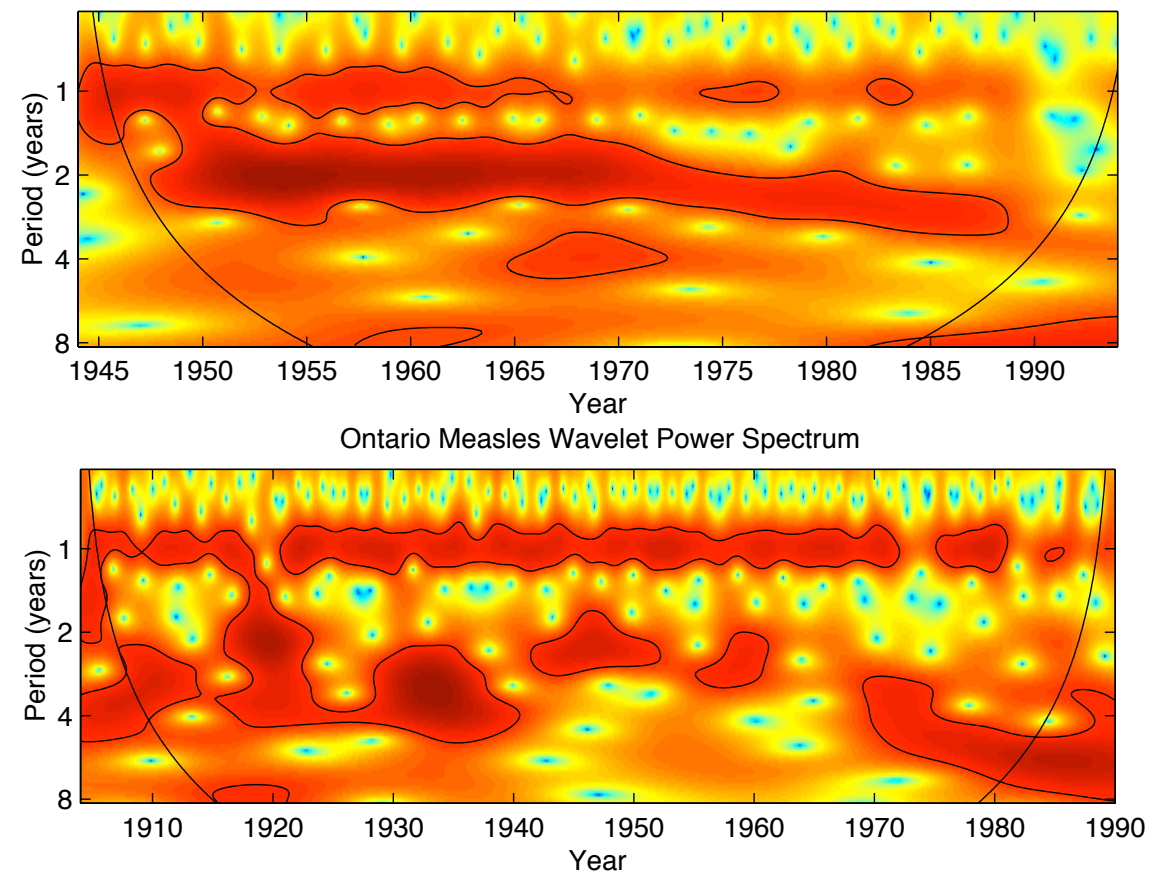

Fig. 7. Wavelet power spectra $[84,85]$ for measles in New York City, USA (top panel), London, England (middle panel) and Ontario, Canada (bottom panel). A complex Morlet wavelet was used. Before computation of the spectra, each time series was log-transormed, its variance normalized to unity, its mean set to zero, and its edges padded with zeros to reduce edge effects [84]. Parabolic lines demarcate the cone of influence (the region of the spectrum in which edge effects are significant). Contours demarcate the $95 \%$ confidence level, hence regions within the contours denote significant peak frequencies. As of time of publication, Matlab scripts for wavelet analysis can be downloaded from http://paos.colorado.edu/research/wavelets/. 
of childhood disease dynamics, but their shortcomings leave still other questions unanswered. In an effort to arrive at a more complete understanding, researchers have developed new models, characterized their properties more completely, and sought closer agreement with data. Today there remain unresolved issues and new questions that continue to attract the attention of mathematicians. One example of an area of continuing research is the influence of spatial structure upon childhood disease dynamics and control [10, 86, 85].

Part of this continuing development is also due to the emergence of new concepts and new techniques of analysis, which often originate in other fields and are then applied to childhood diseases. As new concepts emerge, modellers begin to ask new questions about familiar epidemiological systems, such as: 'Are measles dynamics chaotic?'. Modellers have also applied new methods in time series analysis to childhood disease time series with positive results, as seen in the example of spectral analysis. These new techniques can sharpen understanding of the dynamics behind the time series, and can change the way modellers think about, and discuss, childhood disease dynamics.

These factors, together with the intransigence of childhood diseases, their worldwide health impact, and the relative availability of childhood disease incidence data, suggest that modelling of infectious childhood diseases will continue to be an area of active research for the foreseeable future, and will continue to present new challenges to biomathematicians.

\section{Acknowledgments}

CTB is supported by the Natural Sciences and Engineering Research Council of Canada (NSERC). The spectral analyses that appear in this paper were carried out during the author's tenure as a post-doctoral researcher at McMaster University, 2000-2003, under supervision of Prof. David Earn.

\section{References}

1. P. Bonanni. Demographic impact of vaccination: a review. Vaccine, 17:S120S125, 1998.

2. CDC. Progress toward Poliomyelitis eradication-Nigeria, January 2003-March 2004. MMWR Weekly, 53(16):343-346, 2004.

3. F. Fenner, D. A. Henderson, I. Arita, Z. Jezek, and I. D. Ladnyi. Smallpox and Its Eradication. World Health Organization, Geneva, 1988.

4. F.E. Andre. Vaccinology: past achievements, present roadblocks and future promises. Vaccine, 21:593-595, 2003.

5. World Health Organization. Fact sheet \# 286, March 2005.

6. E. J. Gangarosa, A. M. Galazka, C. R. Wolfe, L. M. Phillips, R. E. Gangarosa, E. Miller, and R. T. Chen. Impact of anti-vaccine movements on pertussis control: the untold story. The Lancet, 351:356-361, 1998. 
7. J.P. Baker. The pertussis controversy in Great Britain, 1974-1986. Vaccine, 21:4003-4010, 2003.

8. V. A. Jansen, N. Stollenwerk, H. J. Jensen, M. E. Ramsay, W. J. Edmunds, and C. J. Rhodes. Measles outbreaks in a population with declining vaccine uptake. Science, 301:804, 2003.

9. B. T. Grenfell and J. Harwood. (meta)-population dynamics of infectious diseases. Trends in Ecology and Evolution, 12:395-399, 1997.

10. D. J. D. Earn, P. Rohani, and B. T. Grenfell. Persistence, chaos and synchrony in ecology and epidemiology. Proc. R. Soc. Lond. B, 265:7-10, 1998.

11. D. J. D. Earn, P. Rohani, B. M. Bolker, and B. T. Grenfell. A simple model for complex dynamical transitions in epidemics. Science, 287:667-670, 2000.

12. C. T. Bauch and D. J. D. Earn. Transients and attractors in epidemics. Proc. R. Soc. Lond. B, 270:1573-1578, 2003.

13. P. E. M. Fine. The interval between successive cases of an infectious disease. Am. J. Epidemiol., 158:1039-1047, 2003.

14. M. B. Priestley. Spectral Analysis and Time Series. Academic Press, London, 1981.

15. R. M. Anderson, B. T. Grenfell, and R. M. May. Oscillatory fluctuations in the incidence of infectious disease and the impact of vaccination: Time series analysis. J. Hyg. Camb., 93:587-608, 1984.

16. S. Hodder and E. Mortimer. Epidemiology of pertussis and reactions to pertussis vaccine. Epidem. Rev., 14:243-267, 1992.

17. H.R. Babad, D.J. Nokes, N.J. Gay, E. Miller, P. Morgan-Capner, and R.M. Anderson. Predicting the impact of measles vaccination in England and Wales: model validation and analysis of policy options. Epidemiol. Infect., 114:319-344, 1995.

18. New Zealand Ministry of Health. Modelling measles. predicting and preventing measles epidemics in New Zealand: application of a mathematical model, 1998.

19. R. M. Anderson and R. M. May. Infectious Diseases of Humans. Oxford Univ. Press, Oxford, 1991.

20. O. Diekmann and J. A. P. Heesterbeek. Mathematical epidemiology of infectious diseases. John Wiley \& Sons, 2000.

21. H. E. Soper. The interpretation of periodicity in disease prevalence. J. Roy. Stat. Soc., 92:34-73, 1929.

22. M. S. Bartlett. Deterministic and stochastic models for recurrent epidemics. Proc. of the Third Berkeley symposium on mathematical statistics and probability, 4:81-108, 1956.

23. D. Green. Self-oscillation for epidemic models. Math. Biosci., 38:91-111, 1978.

24. H. L. Smith. Periodic solutions for a class of epidemic equations. Math. Anal. Appl., 64:467-79, 1978.

25. D. Schenzle. An age-structure model of pre- and post-vaccination measles transmission. IMA J. Math. Appl. Med. Biol., 1:169-191, 1984.

26. H. W. Hethcote. Optimal ages of vaccination for measles. Mathematical Biosciences, 89:29-52, 1988.

27. C. Castillo-Chavez, H. W. Hethcote, V. Andreaen, S. A. Levin, and W. M. Liu. Epidemiological models with age structure, proportionate mixing, and crossimmunity. Journal of Mathematical Biology, 27:233-258, 1989.

28. M. J. Keeling and B. T. Grenfell. Disease extinction and community size: modeling the persistence of measles. Science, 275:65-67, 1997. 
29. D. Greenhalgh. Analytic results on the stability of age-structured epidemic models. IMA J. Math. Appl. Med. Biol., 4:109-144, 1987.

30. H. R. Thieme. Persistence under relaxed point-dissipativity (with an application to an endemic model). SIAM J. Math. Anal., 24:407-435, 1993.

31. H. W. Hethcote and P. van den Driessche. An SIS epidemic model with variable population size and a delay. J. Math. Biol., 34:177-194, 1996.

32. H. W. Hethcote. An age-structured model for pertussis transmission. Math. Biosci., 145:89-136, 1997.

33. H. W. Hethcote. Oscillations in an endemic model for pertussis. Can. App. Math. Quart., 6:61-88, 1998.

34. H. W. Hethcote and P. van den Driessche. Two SIS epidemiologic models with delays. J. Math. Biol., 40:3-26, 2000.

35. F. Brauer. Models for the spread of universally fatal diseases. J. Math. Biol., 28:451-462, 1990.

36. Z. Feng and H. R. Thieme. Endemic models with arbitrarily distributed periods of infection II: fast disease dynamics and permanent recovery. SIAM J. Appl. Math., 61:983-1012, 2000.

37. B.T. Grenfell, O.N. Bjornstad, and B.F. Finkenstadt. Dynamics of measles epidemics: scaling noise, determinism, and predictability with the TSIR model. Ecological monographs, 72:185-202, 2002.

38. W. London and J. A. Yorke. Recurrent outbreaks of measles, chickenpox and mumps. I. Seasonal variation in contact rates. Am. J. Epidem., 98(6):469-482, 1973.

39. J. A. Yorke, N. Nathanson, G. Pianigiani, and J. Martin. Seasonality and the requirements for perpetuation and eradication of viruses in populations. $\mathrm{Am}$. J. Epidem., 109:103-123, 1979.

40. I. B. Schwartz and H. L. Smith. Infinite subharmonic bifurcation in an SEIR epidemic model. J. Math. Biol., 18:233-253, 1983.

41. K. Dietz and D. Schenzle. Mathematical models for infectious disease statistics, pages 167-204. Springer Verlag, New York, 1985.

42. H.W. Hethcote and S.A. Levin. Periodicity in epidemic models. In S.A. Levin, T.G. Hallam, and L.J. Gross, editors, Biomathematics, volume 18, pages 193211. Springer-Verlag, Berlin-Heidelberg-New York, 1989.

43. O. Diekmann, H. Metz, and H. Heesterbeek. The legacy of Kermack and McKendrick. In D. Mollison, editor, Epidemic models: their structure and relation to data, pages 95-118. Cambridge University Press, 1995.

44. H. W. Hethcote. The mathematics of infectious diseases. SIAM Review, 42:599 $653,2000$.

45. F. Brauer and P. van den Driessche. Some directions for mathematical epidemiology. Fields Institute Communcations, 36:95-112, 2003.

46. D. Bernoulli. Essai d'une nouvelle analyse de la mortalite causee par la petite verole. Mem. Math. Phys. Acad. R. Sci. Paris, pages 1-45, 1766.

47. K. Dietz and J. A. P. Heesterbeek. Bernoulli was ahead of modern epidemiology. Nature, 408:513-514, 2000.

48. K. Dietz and J. A. P. Heesterbeek. Daniel Bernoulli's epidemiological model revisited. Mathematical Biosciences, 180:1-21, 2002.

49. W. Farr. Progress of epidemics. Second report of the Registrar General of England, pages 91-8, 1840.

50. W. H. Hamer. Epidemic disease in England. The Lancet, i:733-739, 1906. 
51. J. Brownlee. Statistical studies in immunity: the theory of an epidemic. Proc. Roy. Soc. Edn., 26:484-521, 1906.

52. R. Ross. Report on the prevention of malaria in Mauritius. London, 1908.

53. R. Ross. The prevention of malaria (2nd ed.). Murray, London, 1911.

54. R. Ross and H.P. Hudson. An application of the theories of probably to the study of a priori pathometry, III. Proc. R. Soc, A93:225-240, 1917.

55. W. O. Kermack and A. G. McKendrick. Contributions to the mathematical theory of epidemics I. Proc. Roy. Soc. Lond., 115:700-721, 1927.

56. W. O. Kermack and A. G. McKendrick. Contributions to the mathematical theory of epidemics II. Proc. Roy. Soc. Lond., 138:55-83, 1932.

57. W. O. Kermack and A. G. McKendrick. Contributions to the mathematical theory of epidemics III. Proc. Roy. Soc. Lond., 141:94-112, 1933.

58. A. Hirsch. Handbook of Geographical and historical pathology (volume I). 1883.

59. W. O. Kermack and A. G. McKendrick. Contributions to the mathematical theory of epidemics I-III. Bull. Math. Biol., 53:33-118, 1991.

60. M. S. Bartlett. The critical community size for measles in the United States. J. Roy. Statist. Soc., 123:37-44, 1960.

61. M. S. Bartlett. Stochastic population models in ecology and epidemiology. Methuen, London, 1960.

62. S. F. Dowell. Seasonal variation in host susceptibility and cycles of certain infectious diseases. Emerging infectious diseases, 7:369-374, 2001.

63. P. E. M. Fine and J. A. Clarkson. Measles in England and Wales-I: An analysis of factors underlying seasonal patterns. Int. J. Epidemiol., 11:5-14, 1982.

64. B. Finkenstadt and B. T. Grenfell. Time series modelling of childhood infectious diseases: A dynamical systems approach. J. Roy. Stat. Soc. C, 49:187-205, 2000.

65. N. M. Ferguson, D. J. Nokes, and R. M. Anderson. Dynamical complexity in age-structured models of the transmission of measles virus. Math. Biosci., 138:101-130, 1996.

66. E. B. Wilson and J. Worcester. The law of mass action in epidemiology. Proc. Natl. Acad. Sci., 31:24-34, 1945.

67. E. B. Wilson and J. Worcester. The law of mass action in epidemiology II. Proc. Natl. Acad. Sci., 31:109-116, 1945.

68. H. W. Hethcote, H. W. Stech, and P. van den Driessche. stability analysis for models of diseases without immunity. J. Math. Biol., 13:185-198, 1981.

69. W. Liu, S. A. Levin, and Y. Iwasa. Influence of nonlinear incidence rates upon the behavior of SIRS epidemiological models. J. Math. Biol., 23:187-204, 1986.

70. W. Liu, H. W. Hethcote, and S. A. Levin. Dynamical behavior of epidemiological models with nonlinear incidence rates. J. Math. Biol., 25:359-380, 1987.

71. P. Rohani, M. J. Keeling, and B. T. Grenfell. The interplay between determinism and stochasticity in childhood diseases. Am. Nat., 159:469-481, 2002.

72. M. J. Keeling, P. Rohani, and B. T. Grenfell. Seasonally forced disease dynamics explored as switching between attractors. Physica D, 148:317-335, 2002.

73. R. M. May. Simple mathematical models with very complicated dynamics. Nature, 261:459-467, 1976.

74. W. M. Schaffer and M. Kot. Nearly one dimensional dynamics in an epidemic. J. Theor. Biol., 112:403-427, 1985.

75. L. F. Olsen and W. M. Schaffer. Chaos versus noisy periodicity: Alternative hypothesis for childhood epidemics. Science, 249:499-504, 1990.

76. M. Kot, D. J. Graser, G. L. Truty, W. M. Schaffer, and L. F. Olsen. Changing criteria for imposing order. Ecol. Modelling, 43:75-110, 1988. 
77. W. M. Schaffer. Order and chaos in ecological systems. Ecology, 66:93-106, 1985.

78. L. F. Olsen, G. L. Truty, and W. M. Schaffer. Oscillations and chaos in epidemics: A nonlinear dyamics study of six childhood diseases in Copenhagen, Denmark. Theor. Pop. Biol., 33:344-370, 1988.

79. D. A. Rand and H. B. Wilson. Chaotic stochasticity: a ubiquitous source of unpredictability in epidemics. Proc. R. Soc. Lond. B, 246:179-184, 1991.

80. G. Sugihara, B. T. Grenfell, and R. M. May. Distinguishing error from chaos in ecological time series. Phil. Trans. R. Soc. Lond. B, 330:235-251, 1990.

81. S. Ellner, A. R. Gallant, and J. Theiler. Detecting nonlinearity and chaos in epidemic data. In D. Mollison, editor, Epidemic models: their structure and relation to data, pages 229-247. Cambridge University Press, 1995.

82. B. T. Grenfell, A. Kleczkowski, S. P. Ellner, and B. M. Bolker. Measles as a case study in nonlinear forecasting and chaos. Phil. Trans. R. Soc. Lond. A, 348:515-530, 1994.

83. W. M. Schaffer. Can nonlinear dynamics elucidate mechanisms in ecology and epidemiology? IMA J. Math. Appl. Med. Biol., 2:221-252, 1985.

84. C. Torrence and G. P. Compo. A practical guide to wavelet analysis. Bull. Amer. Meteor. Soc., 79:61-78, 1998.

85. B. T. Grenfell, O. N. Bjornstad, and J. Kappey. Travelling waves and spatial hierarchies in measles epidemics. Nature, 414:716-723, 2001.

86. P. Rohani, D. J. D. Earn, and B. T. Grenfell. Opposite patterns of synchrony in sympatric disease metapopulations. Science, 286:968-971, 1999. 



\section{Index}

$R_{0}, 5$

Age Structure, 10

Attractors, 12, 13

Bartlett, M.S., 10

Basic reproductive number, 5

Bernoulli, D., 6

Brownlee, J., 7

Chaos, 12

Chickenpox, 3, 12

Davidson, A., 7

Dynamical transitions, 13

Hamer, W.H., 7

Hirsch, A., 7

Historical development of models, 6

Incidence terms, 11

Incidence time series, 2, 3

Infectious period, 11

Interepidemic interval, 2

Kermack, W.O., 7

Latent period, 11
McKendrick, A.G., 7

Measles, 3, 12

Monte Carlo models, 10

Polio, 1

Power Spectra, 14

Recurrence, 2, 3, 6

Rubella, 3, 12

Seasonality, 10, 12

SEIR model, 5

SIR model, 4

Smallpox, 1, 6

Soper, H.E., 8

Stability analysis, 5

Stochastic models, 10

Stochasticity, 9

Strain structure, 11

Time delays, 12

Vaccination policy, 1, 2

Wavelet analysis, 16

Whooping cough, 3, 12 
